\title{
LA TECNOCRACIA COMO FORMA DE CONSENSO. EVIDENCIAS A PARTIR DEL CASO DEL SISTEMA DE TRANSPORTE PÚBLICO DE SANTIAGO
}

\author{
Andrea Gartenlaub (gartenlaub@gmail.com) \\ Universidad de Santiago, Chile
}

El artículo busca demostrar cómo el conocimiento técnico se transformó en una herramienta de construcción de consenso en la discusión de la política pública del Sistema de Transporte Público de Santiago. Se argumenta que dicho consenso se construyó en base a imaginarios sociales compartidos los cuales se manifiestan en los discursos de la autoridad y en la pauta de los medios de prensa escrita.

Palabras clave: política pública; Transantiago; consenso; tecnocracia.

\section{TECHNOCRACY AS A FORM OF CONSENSUS. EVIDENCE FROM SANTIAGO'S PUBLIC TRANSPORTATION SYSTEM}

The article seeks to demonstrate how in discussions over Santiago's public transportation system technical knowledge was transformed into a consensus-building tool. It is argued that a consensus was built on the basis of shared social imaginaries, reflected in the discourse of officials and in the written press.

Keywords: public policy; Transantiago; consensus; technocracy. 


\section{INTRODUCCIÓN ${ }^{1}$}

Este trabajo se centra en el análisis de la etapa de formulación de la política pública del Sistema de Transporte Público de la ciudad de Santiago, Transantiago ${ }^{2}$. Bajo este escenario se analizó el concepto de consenso social y cómo éste se reflejó en la agenda de los medios de prensa. A partir de distintas evidencias se presenta la hipótesis de que el conocimiento técnico se alzó como una herramienta de construcción de consenso en la discusión de esta política pública. Se establece que este acuerdo se construyó en base a imaginarios sociales compartidos, los cuales se reflejaron en el discurso de la autoridad y en la pauta de los medios de prensa escrita, irguiéndose este conocimiento tecnocrático como el discurso público hegemónico. Se concluye que la dinámica de consenso, que fue establecida como herramienta para sobrellevar las coyunturas en los primeros gobiernos de la Concertación, se amplió también hacia otros ámbitos, permeando la sociedad chilena más allá de las controversias políticas.

La hipótesis de trabajo se construyó a partir de la evidencia analizada en base a entrevistas a actores, bibliografía y periódicos, y cómo éstos fueron conformando un discurso público proclive a la modernización e impronta tecnocrática de los gobiernos de centro-izquierda. En otras palabras, se construyó un imaginario social que fue la base y la justificación del discurso gubernamental de las políticas públicas. El exitoso establecimiento de este consenso entre gobierno, actores sociales y medios permitió que éste no se alterara por la demanda de los gremios de transporte, principales opositores a esta modernización, sino, por el contrario, se transformara en un disenso con escaso apoyo público. El apoyo ciudadano al Transantiago se mostró amenazado cuando el sistema se vio colapsado en su difícil inicio. Sin embargo, la idea modernizadora se mantuvo inalterable.

1 Este trabajo ha sido posible gracias al aporte de la Comisión Nacional de Investigación Científica y Tecnológica de Chile (CONYCIT) en el proyecto Fondecyt 1080322: "El Proceso de Políticas Públicas en Chile. Análisis de los casos de la Reforma y Modernización de la Gestión Pública, Plan Auge-Ges y Sistema de Transporte Público Transantiago" (2008-2010). Cualquier error u omisión es de exclusiva responsabilidad del autor.

2 Transantiago fue una política pública formulada durante la administración del Presidente Ricardo Lagos (2000-2006) e implementada en el primer semestre del año 2007, durante el gobierno de Michelle Bachelet (2006-2010). 
Durante el proceso de investigación, el análisis de la prensa escrita se transformó en la herramienta para visibilizar las ideas-fuerza de los actores sociales frente a esta política pública, y de paso demostrar su baja capacidad crítica frente a temas de política pública.

El marco teórico utilizado en esta investigación incluyó con conceptos de distintas áreas, como el estudio de la opinión pública, sociología y ciencia política. Y si bien existen otros trabajos que han abordado el tema del Transantiago (Maillet, 2008; Jouffe y Lazo, 2010) desde esta perspectiva constructivista, se cree que este texto puede aportar nuevos antecedentes, desde un acercamiento cualitativo para el estudio de un caso.

Metodológicamente se optó por un estudio de caso, ya que éste se utiliza cuando la pregunta de investigación busca entender el "cómo" o "por qué" de un fenómeno; y cuando la investigación busca indagar en un hecho que tiene contornos no evidentes, es decir, cuando los límites entre el fenómeno y el contexto son difusos (Yin, 1994: 1).

El análisis de los datos se realizó de acuerdo al método de Grounded Theory (Teoría anclada o Teoría fundamentada). Este es un método creado por Glaser y Strauss (1967) que se utiliza para estudios cualitativos, basado en la inducción analítica, que permite generar criterios de codificación de los contenidos de las entrevistas y, a partir de ello, generar explicaciones acerca del caso en estudio. El método comienza con el análisis de los datos hasta construir una teoría explicativa del fenómeno. Para ello mezcla el análisis de contenido clásico, agrupando los datos en categorías analíticas, orientadas a descubrir propiedades que permitan comprender el fenómeno estudiado (Glaser y Straus, 1967, citado en Valles 2007).

Los datos analizados en este trabajo provienen de tres fuentes:

- Treinta entrevistas en profundidad realizadas durante el año 2010 a actores relevantes dentro del proceso de formulación de la política pública del Transantiago. Los entrevistados fueron elegidos de acuerdo a su participación en alguna etapa del período analizado. La identificación de los entrevistados se realizó de acuerdo a los criterios de muestreo teórico o intencionado. Las entrevistas fueron realizadas a través de un cuestionario semi-estructurado. 
- Análisis de las noticias sobre el Transantiago en los principales periódicos publicados del país durante el período del año 2000 al 2008. Se seleccionaron los medios de mayor circulación nacional: El Mercurio, La Segunda, La Tercera, La Nación, La Hora, Punto Final. Este análisis permitió identificar los rasgos públicos de los actores dentro de la discusión política. La metodología usada se basó en la revisión de las publicaciones directamente asociadas al tema, en las áreas de cobertura noticiosa de las secciones Nacional, Política, Económica y Editorial. Los recortes de prensa fueron seleccionados desde el archivo del Centro de Documentación Política del Instituto de Ciencia Política (CEDOP) de la Facultad de Historia, Geografía y Ciencia Política de la Pontificia Universidad Católica de Chile durante los meses de julio a septiembre de 2010.

- La revisión de material y documentos elaborados por técnicos de los órganos públicos, como también investigadores y académicos interesados en el tema. Esta revisión incluyó los discursos y presentaciones públicas de autoridades asociadas a esta política pública. La revisión documental buscó identificar conceptos claves en el proceso de construcción de la política pública.

\section{LOS IMAGINARIOS SOCIALES EN EL DESARROLLO DE UNA POLÍTICA PÚBLICA}

Para entender el proceso de construcción social se partirá por el concepto de imaginario desarrollado por el francés Cornelius Castoriadis. Este autor propone que las creaciones sociales, instituciones, leyes, o en este caso, una política pública, no pueden explicarse fuera de su contexto, debido a que sólo en él cobran sentido sus normas y, en especial, sus propios símbolos y significaciones. "El mundo social [está] constituido y articulado en función de un sistema de significaciones, y estas significaciones existen, una vez constituidas, al modo de lo que hemos llamado lo imaginario efectivo (o lo imaginado). No es sino en relación a estas significaciones como podemos comprender, tanto la elección que cada sociedad hace de su simbolismo institucional, como los fines a los que subordina la funcionalidad'. (Castoriadis, 2010: 236). ¿Por qué utilizar como punto de partida el concepto de imaginario social para analizar el sentido del Transantiago en el discurso público? 
La elección se debe principalmente, a un hecho específico: la cadena de decisiones que estuvo tras la implementación de esta política.

La gran mayoría de los entrevistados expresan que la voluntad que estuvo detrás del impulso presidencial de reestructurar el sistema de transporte público de Santiago, obedeció -más que a diagnósticos técnicos o presiones ciudadanas- , a una decisión previa basada en un sistema de creencias, en un imaginario social, acerca de lo que debía ser una política pública: la modernización.

Son varios los entrevistados que coinciden en señalar que fue el Presidente Ricardo Lagos quien impulsó una reforma que debía estar en consonancia con la visión de un Chile moderno, es decir, dentro de una trayectoria que deseaba poner a la nación en el horizonte de un país desarrollado, del primer mundo. Así, en palabras de un ex asesor del Ministerio de Transporte, "Creo que tiene que ver con el ámbito del desarrollo de la sociedad por sí misma y, en segundo lugar, la impronta, o el sello que tuvo el entonces Ministro Lagos al impulsar un plan de modernización y también de mejorar la infraestructura en distintas áreas. En obras civiles y en transporte, por eso vinieron dos proyectos emblemáticos cuando fue Presidente: el Transantiago y también la recuperación del sistema de ferrocarriles hacia el sur".

Más allá del hecho de que la decisión de transformar el transporte estuviera dentro del programa de gobierno de Ricardo Lagos, se debe recordar que el impulso modernizador del Estado, y en especial de su infraestructura, fue uno de los rasgos distintivos de los gobiernos de la coalición de centro-izquierda, Concertación de Partidos por la Democracia desde su llegada al poder en 1990, y donde el propio Ricardo Lagos tuvo un rol relevante como Ministro de Obras Públicas en el gobierno de Eduardo Frei Ruiz-Tagle (1994-2000).

En términos prácticos la imposición de los gobiernos de Frei Ruiz-Tagle y, en su continuidad, con Lagos, para crear nueva infraestructura en carreteras, puertos, aduanas y modernizar servicios del Estado, se debió a:

a) La necesidad de darle curso a una demanda, tras la postergación, durante los años ochenta, de la construcción de infraestructura de conexión y obras, y también, 
b) Crear una asociación entre actores públicos y privados, es decir, un modelo de política pública, que se llamó sistema de concesiones ${ }^{3}$.

No obstante en términos ideológicos, o de imaginarios sociales, las modernizaciones también se pueden explicar como una forma eficiente de combinar el régimen democrático con tecnocracia. Para Silva (2009), el caso chileno es especial, ya que en él la democracia coexiste con la tecnocracia de forma exitosa y este hecho se debe principalmente "a la necesidad de mantener el modelo económico y por el proceso de aprendizaje político vivido por la élite” (citado en Dávila 2010: 208). Dicho de otra forma, para la Concertación su tarea programática fue la de encontrar el punto de equilibrio, que pudiera superar la lógica de chorreo impuesta por el régimen militar, y en su reemplazo "reconocer el rol y la responsabilidad del Estado en la economía, de su papel regulador y orientador en las grandes líneas de una estrategia de desarrollo" (Valle, 2009: 242).

Lo anterior coincide con la visión del ex ministro de Obras Públicas del gobierno del Presidente Lagos, Carlos Cruz, al recordar que los inicios del afán modernizador de los gobiernos de la Concertación se enmarcaron dentro de la propuesta estratégica de la coalición. "La política fue liderada por el entonces Ministro de Obras Públicas, Ricardo Lagos, y consistió básicamente en enfatizar la necesidad de que el país debía contar con una infraestructura adecuada para cumplir cabalmente sus orientaciones estratégicas en materias económicas: por una parte, estar en condiciones de enfrentar los desafíos de la globalización, en tanto se asumía que era en ese marco que se llevaría adelante el proceso de desarrollo nacional, y por otra, dotar a los habitantes del país de una base mínima común en infraestructura" (Cruz, 2009: 196).

En este sentido, parece obvia la decisión de Ricardo Lagos por modernizar el transporte público, ya que sus antecesores (especialmente Frei Ruiz-Tagle) optaron por otras reformas en este ámbito. Y aunque su programa de gobierno exhibió el slogan "Crecer con igualdad" - muy marcado por la agenda social- también mostró un claro acento en las áreas de la infraestructura, manteniendo el impulso modernizador como parte de la retórica de su gobierno, y alimentando el imagi-

3 Data de 1991 y el encargado de revisar la Ley Orgánica del Ministerio de Obras Públicas fue el Ministro Carlos Hurtado, que envió al Parlamento la Ley de Concesiones. Con ello, se crea el Departamento de Concesiones dependiente de la Dirección General de Obras Públicas. 
nario social sobre la necesidad de crear una política eficiente para desarrollar un país moderno, acorde con los tiempos.

A partir del diagnóstico de retraso se infiere la necesidad de modernización, un término que aparece vinculado al enfoque desarrollista latinoamericano, y que, por otro lado, también tiene una larga historia en la cultura política chilena. La búsqueda del paradigma de la modernización por parte de los gobiernos chilenos no es de ninguna manera exclusiva de los gobiernos de de la Concertación, ya que se puede rastrear desde el período del Frente Popular, desde 1938 a 1952, donde "comienza una etapa sólida de reformas sociales y crecimiento industrial con políticas destinadas a generar una base de infraestructura para la producción y un sistema de protección social para la clase obrera y media" (Garretón, 2007).

Haciendo un recuento histórico dentro del contexto latinoamericano, se debe destacar dentro de estos esfuerzos modernizadores a las reformas de las decadas 50 y 60, de la Administración para el Desarrollo, iniciada para superar los rasgos de una administración patrimonialista, tradicionalista con rasgos paternalistas, que derivó en una administración de corte racional y burocrática donde se establecen relaciones de orden funcional, que tuvo al Estado como su principal protagonista al ser el orientador y promotor de las políticas y asumir directamente la producción de bienes y servicios. Posteriormente, desde mediados de la década de 1980 los intentos modernizadores se reflejaron en los preceptos de la Reforma del Estado. Ésta se dio en un contexto político de cambio con las incipientes democratizaciones; con incertidumbre y presiones en lo económico con la crisis de la deuda externa; mientras de forma paralela, en el plano internacional comenzó a visualizarse el proceso de globalización financiera y comunicacional. Se sumaron a ello, los ajustes estructurales impuestos por las agencias multilaterales, lo que conformó un contexto que impulsó en la administración pública a la adopción de estrategias basadas en el New Public Management, que buscó mejorar la eficiencia de los aparatos administrativos (Cunill, 1997: 225).

En los noventa, con la democracia ya recuperada se inserta la idea de modernización del aparato público, con la reforma a la gestión pública realizada bajo el gobierno de Eduardo Frei-Ruiz Tagle. Tras ello, en un nuevo gobierno, se mantiene la misma lógica y retórica, la que establece el cambio del sistema de transporte de la ciudad de Santiago, el cual se había venido trabajando en las administraciones anteriores. Este proceso se inicia con un diagnóstico: 
Hay una constatación de que el sector está en manos de actores identificados con una situación de subdesarrollo, [de ello] se deduce la necesidad de cambio, y más específicamente, de ruptura. Así, el Presidente Lagos, en una visita a las obras de extensión del Metro, califica el proceso de modernización del transporte público de revolución más grande que existe en la historia de Chile en materia de infraestructura. [Esto] hace visible la inscripción en una trayectoria histórica que culmina en los proyectos del Bicentenario, y por otra parte, la palabra revolución es un marcador de la ambición de la reforma de arrasar con el pasado (Maillet, 2008).

Al referirse de forma específica al transporte, el programa de gobierno de Ricardo Lagos expresa el deseo de reforma en el sector:

Modernizaremos el transporte urbano de Santiago. Mejoraremos la seguridad, tiempos de viaje y calidad del transporte. Impulsaremos la construcción de vías exclusivas para vehículos de transporte público. Insistiremos en la profesionalización de los choferes y el mejoramiento de sus condiciones de trabajo. En Santiago se invertirá en seis corredores principales con una extensión total de 60 kilómetros y se ampliará la red del Metro abarcando 25 kilómetros en total. Se reestructurarán los recorridos y contarán con sistemas tarifarios integrados entre buses y con el Metro. Con un boleto inteligente se podrá viajar en los distintos servicios. Fomentaremos la renovación del parque de buses introduciendo tecnologías no contaminantes. Se implementarán acciones decididas de reducción de los accidentes de tránsito. Se adecuarán los espacios públicos para hacer más atractivas y seguras las caminatas y facilitar el uso de la bicicleta. Diseńaremos mecanismos que aseguren el financiamiento y la fiscalización del Plan, en el entendido que Santiago tendrá que hacerse cargo de sus costos (Lagos, 1999). 


\section{CONSENSO Y TÉCNICA, CLAVES PARA LA GOBERNABILIDAD}

Si por un lado el discurso del gobierno se enmarca dentro de lo que fue el ideario programático de la coalición de centro izquierda, cómo podemos explicar el alto grado de consenso logrado para estas reformas. ¿Sólo se debió a un consenso social pactado entre las autoridades y las elites?, ¿o éste acaso permeó hacia la opinión pública, por ende, a los medios de comunicación?

En primer lugar, entendemos por consenso la definición entregada por Giacomo Sani como "la condición determinada del sistema de creencias de una sociedad, que existe cuando entre los miembros de una unidad social dada hay acuerdo acerca de principios, valores, normas y también respecto de la deseabilidad de ciertos objetivos de la comunidad y los medios aptos para lograrlos" (citado Riorda, 2006: 19).

Siguiendo este concepto, y en términos operativos, ¿cuál sería el objetivo de un gobierno por mantener el consenso? Si no, mantener, conseguir y gestionar ciertos niveles de adhesión a las decisiones de administración. Según Elizalde (2006: 153), "el consenso es más difícil de alcanzar en un entorno de mayor complejidad social; la complejidad social se transforma en complejidad comunicativa, en tanto la sociedad actual puede ser definida como sociedad mediática (sociedad de la información) y una sociedad con índices más elevados de protesta y de participación social en el escenario público (...) la complejidad social crece en la medida que la protesta y la participación se retroalimentan y se fortalecen mutuamente".

En este sentido lo realmente novedoso es constatar cómo un mecanismo para establecer acuerdos políticos, se traspasa a lo social estableciéndose con una virtud compartida, en este caso, por los medios de prensa locales. Se debe destacar que la dinámica de consenso, fue establecida como herramienta para sobrellevar las coyunturas en los primeros gobiernos después de la transición, no obstante, de alguna forma "la democracia de los consensos" se amplió hacia otros ámbitos, no sólo en las controversias políticas.

Concordamos con Baeza-Rodríguez (2008) para explicar que la dinámica de consenso fue ampliamente usada como un barómetro para medir el grado de gobernabilidad de las administraciones democráticas. Según la autora, 
La noción de gobernabilidad parece, en efecto, desempeñar un papel de discurso ordenador. $\mathrm{Al}$ articular un relato aceptable de democracia moderna donde la capacidad de gobernar no puede sino provenir de un poder tecnocrático y estable, el registro de la gobernabilidad aparenta resolver las contradicciones de la transición democrática chilena. Se minimiza así la deformación del sufragio universal al compararla con las cualidades estabilizadoras y moderadoras del sistema electoral binominal; la desarticulación de la ciudadanía se ve reinterpretada positivamente como la desideologización de la sociedad y como su adhesión a los grandes principios consensuales de la nueva tecnocracia, entendida aquí, a la vez, como el gobierno de la racionalidad técnica y como el borrado de las fronteras entre las profesiones políticas y técnicas (Baeza-Rodríguez, 2008).

La idea señalada en el párrafo anterior parece dar algunas claves para explicar cómo todo un imaginario social sostenido por los pilares de consenso y gobernabilidad, pudo permear hacia otros actores: los políticos, y también la prensa. Una de las respuestas posibles a ese trasvasije es que el conocimiento técnico también puede usarse como herramienta de construcción de realidad. La idea no es nueva, y se ha aplicado en otros campos como la historia de la ciencia y los paradigmas científicos, es así como el uso del concepto controversia socio-técnica ${ }^{4}$ es un fenómeno estudiado por la sociología de la ciencia, el que se entiende como la implementación de innovaciones tecnológicas en la sociedad.

Para estos estudios las tecnologías no obedecen a factores endógenos de la propia ciencia, más bien son el resultado de negociaciones, imposiciones, consensos y traducciones que los posibilitan, instalando el debate respecto a la necesidad de su implementación. Dentro de este enfoque Pinch y Bijker (1989) utilizan la perspectiva de la sociología constructivista para explicar que los artefactos científicos se construyen socialmente. Esta construcción tiene etapas, las cuales son el debate

4 El concepto de controversia socio-técnica aparece en el campo de la sociología como uno de los principios constitutivos del Programa Fuerte de David Bloor (1974) para analizar la construcción de los conocimientos científicos a través de teorías, modelos que deben estudiarse usando el mismo conocimiento que el nuevo paradigma dio por superado y erróneo. Una década más tarde, llega el enfoque de la construcción social de la tecnología (Pinch y Bijker, 1989), que utiliza la sociología constructivista para el estudio social de la tecnología. 
de las alternativas, la estabilización de una alternativa y la clausura del debate con la redefinición del problema que lo originó. Es por ello que podemos pensar que el conocimiento técnico para la implementación de una política, también se encuentra bajo un imaginario social subjetivo que hace que las ideas y sus artefactos tecnológicos, no sólo tengan que ver con aspectos técnicos y objetivos, sino con las creencias y valores de quien los crea.

Al aplicar esta perspectiva a este caso de estudio, más que meros artefactos técnicos, los sistemas de transporte se pueden entender como un dominio en el que actores sociales y no sociales (entiéndase como infraestructura) conforman una red de asociaciones en permanente emergencia; y, que bajo determinadas condiciones, y dada su importancia en el medio social, adquieren capacidad de agencia, de movilizar recursos, y de constituirse en un objeto de controversia (Latour, 2005: 91). En este punto es cuando se hace necesario entender cómo "la idea de modernización" se puede transformar en un discurso dominante que, a través de los procesos sociales y tecnológicos, pueden verse develados como una innovación necesaria para una autoridad, y por ende, para la sociedad.

A partir de estos conceptos se pone en evidencia la importancia que adquieren los arreglos institucionales, y por ende los procesos de negociaciones, y de consensos que configuran la creación de una tecnología, o en este caso específico, la adopción de un nuevo sistema de transporte. En suma, según la perspectiva de las controversias socio-técnicas, la ciencia y los expertos están sociotécnicamente construidos, por lo tanto, las innovaciones serán políticamente impuestas a los ciudadanos.

En este punto, podemos decir que la perspectiva construccionista se acerca bastante a la propuesta denominada coaliciones promotoras de politicas públicas, desarrollada por los norteamericanos Sabatier (1988) y Jenkins-Smith (1993), en la cual se expresa que existen múltiples actores que comparten un set o conjunto de creencias que se traducen en planes de políticas públicas. Este enfoque también da énfasis al rol subjetivo de las "creencias normativas", ya que éstas también pueden constituirse en factores claves para la creación de un plan de política pública.

En una perspectiva similar, pero desde la escuela francesa de las políticas públicas, Jobert (2004) ocupa una reflexión parecida. Su concepto es el de "referenciales": un conjunto de creencias, valores y técnicas que dan forma a la estructura de las políticas públicas. Para Jobert, estos referenciales tienen tres dimensiones: una 
cognitiva, que da elementos de interpretación a los problemas y su solución; una normativa, que define los valores que habría que ocupar en el tratamiento del problema, y una instrumental, que define los principios de acción que deben orientar la acción en función de sus valores (Jobert, 2004:93).

A partir de los enfoques expuestos, la perspectiva constructivista parece ser útil para entender cómo se estructura el "consenso social" dentro de una sociedad, y cómo los arreglos institucionales se presentan necesarios para mantener ciertos estándares de gobernabilidad. En este sentido, el papel de las políticas públicas no escapa a estas negociaciones; más bien, es parte de esta dinámica, imponiendo y siendo parte, desde su formulación, en el sistema de creencias de un gobierno.

\section{3. ¿POR QUÉ ESTAMOS TODOS DE ACUERDO? LA FORMACIÓN DEL CLIMA DE OPINIÓN}

Cuando pensamos en cómo se forma y se construye el consenso, no sólo se debe centrar la atención a las disputas sobre la información técnica, también debemos entender cómo se administra la información en la sociedad. En este punto utilizaremos el concepto de Espiral del Silencio, acuñado por Noelle-Neumann para explicar cómo la opinión pública se transforma en una especie de control social en que los individuos van adaptando una opinión y/o un comportamiento a partir de las actitudes predominantes sobre lo que se considera aceptable. Estas actitudes se pueden asimilar a un set de temas públicos que se instituyen como lo verdaderamente importante, dejando otros ignorados, olvidados, a pesar de que existan. La principal tesis de Noelle-Neumann se basa en la idea de que el conocimiento, al ser público, se legitima, no importando si éste es verdadero o falso. El punto en cuestión es lo que se ha logrado establecer como consenso, o el llamado clima de opinión.

El clima de opinión es la tendencia mayoritaria sobre determinadas opciones, las que son compartidas por gran parte de la población. Según Noelle-Neumann, éste actúa como un fenómeno de contagio ya que la opción mayoritaria se extiende rápidamente por toda la sociedad, y se vuelve un sentimiento hegemónico respecto a un tema. Esta idea explica las dinámicas de los medios de comunicación masiva al imponer sus propios temas (función de agenda), y porqué de entre un universo amplio de temas, sólo se seleccionan unos cuantos, es decir, cómo a 
algunas personas y/o argumentos se les confiere un prestigio o reputación mayor que a otros.

Al utilizar los términos de espiral del silencio (los temas desechados de la agenda) y clima de opinión (lo que una mayoría asume como el pensamiento hegemónico) podemos decir que la construcción de consenso técnico se basa en la idea compartida socialmente de que lo que dice el experto es eficiente, real y sobre todo verdadero.

\section{1 ¿Cómo se construye el consenso?}

Si la información en la sociedad se distribuye a través de ciertas dinámicas, es tarea de los gobiernos conocer y manejar ciertos recursos y repertorios para administrar su información, siendo éste un fenómeno que cada día se vuelve más importante en la comunicación gubernamental, convirtiéndose en un punto complejo y altamente sensible para las administraciones. Para Elizalde (2006:164):

Los problemas de comunicación con los que se encuentran los gobiernos en la actualidad pueden hacer fracasar y desestabilizar sus políticas públicas más eficaces. No sólo porque sean desestabilizados por crisis imprevisibles o por escándalos políticos, financieros, corrupción o incluso sexuales, sino por el efecto erosivo (a mediano plazo) de la falta de consenso motivado por ciertas crisis de reputación que se derivan en pérdidas de credibilidad y de legitimidad, lo que en un plazo más largo produce erosión sobre la confianza básica y sobre la institucionalización.

En el caso del Transantiago, en un primer momento, el gobierno jugó con varias fichas a su favor. Tras la decisión de llevar a cabo la política, el gobierno comenzó a trabajar con la Espiral del Consenso-Disenso (Elizalde, 2006), para instalar un discurso que atrajera a la mayoría de los ciudadanos y también desestabilizara a sus principales oponentes, los gremios de locomoción colectiva, al definirlos como "contrarios a las modernizaciones". Desde la visión de las políticas públicas existe una visión menos contenciosa en la definición de gremios. Por ejemplo, los sindicatos o gremios se unen por razones no políticas como conseguir sueldos más elevados y más seguridad, vale decir, se unen por razones que poco tienen que 
ver con sus funciones o con el proceso de políticas públicas (Lindblom, 2001). Entonces, más que el deseo de influir en las políticas, la unión entre individuos se produce por los beneficios particulares que genera a sus miembros (Olson, 1978). En el caso de los trabajadores, Kingdon (1995) plantea que su participación en las políticas públicas es menos frecuente que en otros grupos y, como consecuencia, como grupo de presión, no serían tan importantes en lo que respecta a la emergencia de la agenda. Su presencia o nivel de presión variaría según el ámbito en que se desarrollan (transporte o salud)" (Gaete, 2010: 22).

Ejemplificando lo que fue la relación gobierno/gremios, podemos ver distintas aristas. Por ejemplo en una entrevista publicada en el diario La Nación, el 26 de julio de 2002, el entonces coordinador del Programa de Transportes, Germán Correa, analizaba el rechazo de los microbuseros a la licitación por recorridos que se realizaría el año 2003.

Ellos dicen que esto no significa oponerse a la modernización que viene, pero si esto no es oponerse no sé lo que es. Vamos a tratar de ayudarlos en este cambio, pero si en la próxima licitación están en la misma actitud de ahora, no van a tener futuro en el sistema de transporte. No vamos a ser nosotros los que los dejemos afuera.

En tanto, los gremios exteriorizaban su disconformidad contra este proceso, debido a que consideraban que éste los excluía incluso con antelación, debido a las altas cuotas de incorporación que se exigían para participar en las licitaciones. En una nota fechada el 20 de marzo de 2004 en el diario La Tercera, se señalaba:

Dirigentes microbuseros presentan recurso contra plan Transantiago. El gremio de los microbuseros interpuso un recurso de protección contra el plan Transantiago, por considerar que trasgrede diversos derechos de los empresarios del sector al imponer bases arbitrarias en los planes de licitación (...) Según el abogado José Hinzpeter, del sector, se están fijando recursos importantes para participar, 500 mil UF. Eso es una garantía mayor que la exigida para poner una financiera o una bolsa de comercio. 
En este escenario, otra de las estrategias del gobierno para instalar el tema en la opinión pública fue la realización de una serie de diagnósticos ${ }^{5}$ referidos a dificultades de índole ambiental, seguridad ciudadana, deficiencia económica y malas prácticas gremiales que provocaba el sistema de transportes, en este sentido:

Transantiago no sólo fue presentado como la solución frente a los problemas de los microbuses sino que también era uno de los medios para convertir a Santiago en una ciudad de clase mundial, moderna y competitiva a nivel internacional. De este modo el discurso que portaba este cambio estigmatizaba el carácter caótico de los microbuses amarillos, indignos de una nación que aspiraba a ser parte de los países en desarrollo (Jouffe y Lazo, 2010).

Por ejemplo, para una ex autoridad regional, el cambio se debía principalmente a la necesidad de velar por la seguridad y salud de los santiaguinos, y de paso llevar a cabo un plan de descontaminación:

\section{Las micros amarillas eran bastante contaminantes y era necesario} hacer una renovación muy significativa de flota para poder cumplir con el Programa de Descontaminación de Santiago ${ }^{6}$. Además el sistema aquel era altamente inseguro en términos de los accidentes, de la seguridad de los conductores y de los mismos usuarios, incluso en temas de delincuencia. Y en el sentido de la cantidad de buses que

5 Para una síntesis véase el trabajo de Guillermo Díaz, Andrés Gómez Lobo y Andrés Velasco (2004), titulado de forma bastante categórica: "Micros en Santiago: De enemigo público a servicio público". En este paper se resume buena parte de los diagnósticos sobre el sistema de transporte capitalino: "El sistema de transporte público de Santiago tiene algunas virtudes, tales como su amplia cobertura y frecuencia. Sin embargo, también lo aquejan serios problemas de congestión, inseguridad, ineficiencia y mala calidad del servicio. Los microbuses también son responsables de buena parte de la contaminación del aire y acústica que padece Santiago. Estos problemas justifican una reforma de fondo. Existe un cierto consenso a nivel técnico sobre los lineamientos generales que debería seguir dicha reforma. El Plan Transantiago, que entrará en vigencia en 2005, pretende optimizar los recorridos, integrar los modos de transporte y las tarifas, crear empresas y modernizar los buses, todo ello mediante un nuevo sistema de licitaciones, que modifique radicalmente el régimen que rige desde comienzos de la década de los noventa”.

6 El Plan de Descontaminación de Santiago (2005) fue una modificación del Plan de Prevención y Descontaminación de la Región Metropolitana. Buscaba evitar los episodios de crisis medioambientales (preemergencias) a través de una serie de medidas como el mejoramiento de los combustibles y la exigencia de las normas de emisión para las actividades productivas que se desarrollaban en la cuenca de Santiago. 
circulaban, con toda la ineficiencia que eso significaba en términos de congestión, tiempos de viajes y costos (ex autoridad regional).

No obstante, respecto a intereses ciudadanos, el transporte público no parecía ser una demanda de primer orden, aunque existía el consenso de que el servicio podía mejorar:

En encuestas que se manejaban en la época, había una nota muy baja para el transporte público. Ahora tampoco podría decir que había una demanda, o sea que habían marchas en la calle por cambiar el sistema. Pero eso, no es la única razón para enfrentar una política pública (Asesor Técnico).

También existió un diagnóstico de orden económico.

Era cierta ineficiencia económica: los choferes ganaban mucha plata para la educación que tenían, o sea claramente un sector donde se pagaba una prima para competir en la calle que era ineficiente, porque no era algo que generara, en mi opinión, un mejoramiento económico, considerando las alternativas que existían (Asesor Técnico).

De forma mayoritaria todas estas consideraciones venían desarrolladas desde el ámbito técnico-académico, y con un claro sesgo en contra del empresariado del sector, siendo éste el punto crítico más utilizado con el sistema. La respuesta, eso sí, era unánime: se debía regular y reestructurar el mercado del transporte:

Había un problema económico básico de externalidad. Y el mercado, en realidad, cuando no funciona, el único que puede tener un rol regulador, es el Estado. Pero no es fácil regular cuando hay miles de empresarios. Es decir, había un empresario por cada microbús. En los países desarrollados había un sistema mucho más estructurado (Ex autoridad del sector).

Confirman esta misma idea crítica acerca de las micros amarillas las versiones de dos expertos en transporte. "Existía una sensación de libertinaje respecto al an- 
tiguo sistema y desde el punto de vista más técnico era un sistema que no tenía cómo mejorar y que solo podía empeorar [...] Era la principal fundamentación para proponer un cambio mayor al sistema de transporte público de Santiago". Agrega otro entrevistado: "El fundamento principal para modificar el sistema de transporte es que el sistema antiguo era un sistema más bien basado en casi una prestación de servicios artesanal".

En síntesis, lo que se explicita en los párrafos anteriores es cómo se fue creando una percepción, un clima de opinión, a través de un discurso sobre cómo se debía encarar esta política: una que debía ser pensada racionalmente, estructurada a través de una política pública de Estado, y principalmente con nuevos actores en el rol de proveedores, los privados. La suma de estos criterios llevó a la autoridad a buscar un amplio repertorio de razones para implementar la medida, y principalmente minimizar los efectos de sus oponentes: los gremios. Entonces, ¿̨cuál fue el discurso público implementado por el gobierno? Un dirigente gremial lo explica:

La vendida de pomada que le hicieron a la gente fue que el sistema de transporte anterior era malo. $Y$ entonces, ellos quisieron hacer una revolución en el transporte desde lo económico, lo cual fracasó. No vieron otro punto de vista que ése. Y se dieron cuenta que un ente fiscal nunca operó en ninguna actividad. Y tuvieron que entrar a meterse lisa y llanamente en términos reales, es decir, en términos operativos. Los mismos bancos ingresaron a un negocio tecnológico que nunca habían estado acostumbrados a hacer y fracasaron. Entonces pastelero a tus pasteles... Hicieron una transformación que no estaba acorde con lo que correspondía hacer en este país, que era un desarrollo paulatino del sistema de transporte.

Los gremios, el año 2000, ya mostraban cierta reticencia y durante el año 2002 fueron abiertamente críticos al sistema. Un ejemplo de la escalada del conflicto entre gobierno y empresarios de la locomoción colectiva, fue la crónica del diario La Tercera del 9 de marzo de 2002:

7 Nota del Editor: "Vender la pomada", es una expresión coloquial utilizada en Chile que quiere decir, convencer a alguien para participar en algún proyecto inviable con el propósito de obtener un beneficio. 
Surgen primeros roces entre Germán Correa y microbuseros. Presidente de los autobuseros replicó en duros térmicos declaraciones del ex ministro sobre mala calidad del servicio. Manuel Navarrete recordó que las condiciones de operación de los buses fueron la consecuencia de las bases del proceso que encabezó el ex secretario de Estado cuando era ministro de Transportes en el gobierno de Patricio Aylwin.

Así es como el tono de enfrentamiento se mantiene con declaraciones del tipo: "No le tengo miedo a los microbuseros", realizadas por el mismo Correa al diario El Mercurio el 4 de agosto de 2002.

Es en este punto cuando los medios parecen adoptar consenso contra los gremios y en pro de la modernización a través de sus notas editoriales. Por ejemplo, el diario La Tercera, el 13 de agosto de 2002 publicaba:

Santiago no puede ser rehén de los microbuseros. Nada justifica el llamado de los empresarios del transporte público a paralizar las máquinas de locomoción colectiva y bloquear las principales avenidas de Santiago como forma de rechazar la reciente licitación realizada por el gobierno. Menos aún cuando el objetivo que las autoridades tienen en mente es la modernización de uno de los gremios peor evaluados por la ciudadanía (...) Este último se ha opuesto a todo aquello que apunte a una modernización y orden de la gestión del transporte público.

Aparentemente el discurso mediático fue el reflejo de lo que los propios expertos (técnicos y principalmente ingenieros en transporte que participaron en el desarrollo del plan) tenían sobre los gremios.

El sistema volvió a hacer crisis, pero más de la perspectiva política, cuando hicieron el famoso paro de los micreros ${ }^{8}$. Y eso fue lo que

8 El paro se realizó el 12 y 13 de agosto de 2002 y fue convocado en protesta por las bases de las licitaciones de recorridos establecidas por el Gobierno. En respuesta, el Ministerio del Interior y la Intendencia de Santiago presentaron querellas contra los presidentes de los gremios, quienes fueron detenidos el mismo 13 de agosto. Los cinco dirigentes: Demetrio Marinakis, Manuel 
probablemente desencadenó la decisión política de introducir un cambio mayor en el sistema. Si uno le preguntaba a mucha gente, todos tenían críticas, pero no eran todas las mismas. Ahora, ciertamente, yo creo que la decisión más política, y que implicaba un cambio de mayor importancia, era atacar el tipo de organización empresarial que existía. O sea una organización que podía ser de empresarios, gremios o carteles ${ }^{9}$. Efectivamente desde el punto de vista económico su comportamiento era más propio de un cartel; desde otro punto de vista, ellos actuaban más parecidos a un gremio. Pero por otro lado, en la actividad cotidiana eran como una familia. Había una cantidad de vínculos que excedía lo puramente corporativo. Había familias enteras metidas en el negocio, por generaciones. Había toda una cultura que era mucho más que una asociación reunida en defensa de ciertos intereses. Por lo tanto, no era un cambio menor. Cualquier cambio de esa estructura, sin duda, era de gran magnitud (experto en Transporte).

Desde el punto de vista de la teoría comunicacional, se puede decir que el gobierno manejó el Transantiago (situándonos siempre en su primera etapa, es decir en la formulación, mucho antes de su implementación) con un modelo de difusión y de marketing. El modelo de difusión se define en cómo controlar los procesos de información que son relevantes para temas de interés público. "Este es un modelo comunicacional que se adapta a la concepción del Estado tecnocrático y a la concepción iluminista clásica que supone que el gobernado deberá estar informado de manera impersonal, fría y objetivamente acerca de lo que sucede" (Elizalde, 2006: 208).

Navarrete, Armando Huerta, Orlando Panza y Marcel Antoine estuvieron detenidos durante 14 días. Posteriormente fueron condenados a tres años de cárcel. No obstante, la pena se cumplió de forma remitida, un año después, en 2003.

9 En vista a lo que fueron sus recursos de acción, los gremios de transporte no sólo actuaron como asociaciones de trabajadores, sino también exhibieron otras formas de acción pública, a la manera de grupo de presión. Citando a Kingdon (1995), podemos señalar que un grupo de presión tiene capacidad de influencia, a través de diferentes mecanismos: persuasión, intimidación y corrupción. La persuasión se dirige a actores dentro del gobierno a través de negociaciones y/o de propagandas, con el objeto de presentar sus ideas, necesidades o requerimientos como acciones orientadas al bien común. La intimidación se expresa a través de hechos o amenazas de fuerza como huelgas, paros o movilizaciones. En casos extremos, la corrupción se establece como el pago de dinero para forzar una decisión política favorable. 
Se puede pensar que fueron éstos los mecanismos que permitieron que los medios fueran altamente receptivos al contenido tecnocrático propuesto por el gobierno, principalmente en la creación de un discurso en contra de los gremios-empresarios de transporte, y que también posibilita explicar cómo fue adoptado sin mayores cuestionamientos por parte de la prensa. Pero ¿cuál fue la razón de tal sumisión al discurso técnico? Al parecer el conocimiento de expertos técnicos fue altamente seductor para los medios, lo que ayudó a imponer este discurso sobre otras consideraciones de orden social, gremial o ciudadano, mostrándose como una forma hegemónica de entender la realidad. De hecho la idea de la sabiduría del experto, por sobre otras formas de conocimiento, fue una constante que se mantuvo durante todo el período estudiado.

\section{MIDIENDO EL CONFLICTO: LA ESPIRAL DEL DISENSO-CONSENSO}

En este punto se analiza el rol de la prensa escrita del período 2000-2008 durante la formulación de la política de transporte público de Santiago. Para ello queremos utilizar un concepto denominado Espiral disenso-consenso usado por Elizalde (2006). Esta idea se basa en la necesidad de crear un clima de opinión o consenso proclive a la autoridad. Para Elizalde los gobiernos, en su función de poder ejecutivo, se encuentran en permanente sobreexposición pública, la que se ve exigida por la sensibilidad social y política, y el aumento de participación social, de diferentes grupos sociales. Por ello, "la comunicación y la política gubernamental están unidas principalmente por el hecho de que el gobierno necesita aumentar o mantener su poder por medio del consenso y de la concertación de voluntades" (Elizalde, 2006: 159).

Para ilustrar un ciclo de consenso y disenso, una herramienta útil es definir cuál fue el nivel de conflictividad de los principales periódicos de circulación nacional, cuáles fueron los actores con más presencia mediática, y los periodos de mayor conflictividad. Describir estas dinámicas permite mostrar con claridad cuál fue la curva ascendente del consenso (período de formulación de la política pública, en los años 2000-2006) y el momento de la crisis, o descenso, en el momento de la implementación del Transantiago (primer semestre de 2007). 
A partir de un corpus compuesto por las noticias acerca del tema, publicadas por los principales periódicos de circulación nacional, se trabajó en conocer cuáles fueron los períodos más conflictivos del proceso, y los actores con mayor presencia en la pauta. Para realizar esta investigación se revisaron 1.265 notas de prensa de los diarios El Mercurio, La Tercera, La Segunda, La Nación y otros medios, durante los años 2000 al 2008. El desglose de las notas de prensa por periódico es el siguiente:

\section{Gráfico 1}

Notas sobre el Transantiago publicadas en los diarios de circulación nacional (2000-2008)

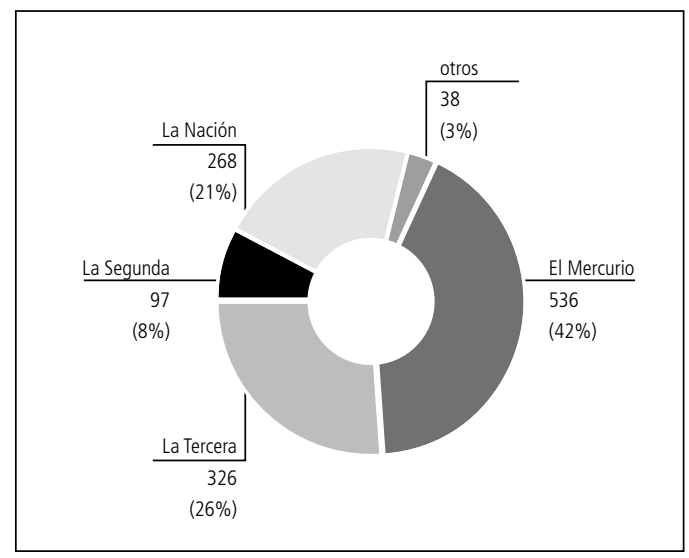

Fuente: Elaboración Propia

Como metodología se llevó a cabo un análisis de contenido básico, a través de una lista de códigos, un code book a través de palabras con énfasis contencioso como Crisis, Deficiencias, Paro, Errores, Dificultades, Ataques, Críticas, entre otras de similar significado. A los títulos y bajadas de cada noticia se les asignó un número de 1 a 4 para definir su grado de conflicto siendo 1 para "grado de conflicto bajo", 2 para "moderado", 3 para "alto" y 4 para "muy alto". A partir de la codificación obtenida se muestra que la conflictividad de las notas de prensa se concentró durante el primer semestre del 2007, fecha coincidente con la puesta en marcha del plan Transantiago, el 10 de febrero de ese ańo (ver cuadro 1). 


\section{Cuadro 1}

Porcentaje del nivel de conflictividad de las notas publicadas sobre el Transantiago por semestres (2000-2008)

\begin{tabular}{|c|c|c|c|c|}
\hline \multirow{2}{*}{ Año por semestre } & \multicolumn{4}{|c|}{ Nivel de conflictividad } \\
\hline & Muy alta & Alta & Moderada & Baja \\
\hline Primer semestre 2000 & $0,50 \%$ & $0,30 \%$ & $0,00 \%$ & $0,70 \%$ \\
\hline Segundo semestre 2000 & $0,20 \%$ & $0,30 \%$ & $0,00 \%$ & $1,40 \%$ \\
\hline Primer semestre 2001 & $0,50 \%$ & $1,20 \%$ & $0,40 \%$ & $2,80 \%$ \\
\hline Segundo semestre 2001 & $0,00 \%$ & $0,00 \%$ & $0,00 \%$ & $0,00 \%$ \\
\hline Primer semestre 2002 & $1,60 \%$ & $0,90 \%$ & $4,00 \%$ & $5,60 \%$ \\
\hline Segundo semestre 2002 & $1,80 \%$ & $2,60 \%$ & $2,20 \%$ & $1,40 \%$ \\
\hline Primer semestre 2003 & $2,00 \%$ & $2,00 \%$ & $1,30 \%$ & $2,80 \%$ \\
\hline Segundo semestre 2003 & $0,40 \%$ & $0,60 \%$ & $0,00 \%$ & $0,70 \%$ \\
\hline Primer semestre 2004 & $4,00 \%$ & $4,00 \%$ & $2,70 \%$ & $4,20 \%$ \\
\hline Segundo semestre 2004 & $0,90 \%$ & $2,30 \%$ & $2,20 \%$ & $6,30 \%$ \\
\hline Primer semestre 2005 & $5,80 \%$ & $2,90 \%$ & $3,60 \%$ & $7,70 \%$ \\
\hline Segundo semestre 2005 & $1,10 \%$ & $0,60 \%$ & $1,80 \%$ & $3,50 \%$ \\
\hline Primer semestre 2006 & $11,00 \%$ & $9,00 \%$ & $5,40 \%$ & $15,50 \%$ \\
\hline Segundo semestre 2006 & $0,50 \%$ & $0,30 \%$ & $0,00 \%$ & $0,70 \%$ \\
\hline Primer semestre 2007 & $58,00 \%$ & $55,50 \%$ & $48,70 \%$ & $38,00 \%$ \\
\hline Segundo semestre 2007 & $0,00 \%$ & $0,00 \%$ & $0,00 \%$ & $0,00 \%$ \\
\hline Primer semestre 2008 & $11,60 \%$ & $17,30 \%$ & $27,70 \%$ & $8,50 \%$ \\
\hline Segundo semestre 2008 & $0,00 \%$ & $0,30 \%$ & $0,00 \%$ & $0,00 \%$ \\
\hline$\%$ Total & $100 \%$ & $100 \%$ & $100 \%$ & $100 \%$ \\
\hline
\end{tabular}

Fuente: Elaboración Propia

Ejemplos de este período hay varios, como la editorial del diario El Mercurio, del 20 de marzo de 2007 titulada: "El drama del Transantiago" que resume el ambiente tras la implementación del servicio.

Luego de más de un mes de funcionamiento, los problemas del Transantiago se han vuelto permanentes para gran parte de los habitantes de Santiago. Incluso los automovilistas, inicialmente favorecidos, ahora enfrentan a una congestión peor. Asimismo, las 
aglomeraciones en el Metro confirman la gravedad de la situación. Esto ha sido admitido por su presidente, quien, con gran coraje, ha llamado a embarazadas, ancianos, nińos y minusválidos a evitarlo, y declarado que el nivel de servicio del transporte público no es aceptable. Y el Ministerio de Transportes -en una muestra más de la presión que pesa sobre él- anunció importantes modificaciones a los recorridos, sin antes comunicarlas a la empresa contratada para informar a los usuarios del Transantiago.

En tanto, el diario La Tercera, en la misma fecha, 22 de marzo de 2007, describía la polarización establecida entre gobierno y oposición tras la problemática implementación del sistema:

Crece tensión por Transantiago: Comité se reúne en La Moneda y Alianza emprende nueva arremetida. En la sede de gobierno, el subsecretario de Transportes Danilo Núñez, dijo que su equipo sigue contando con la confianza de la Presidenta y aseguró que: tenemos más fuerza que nunca para seguir adelante y no le tenemos miedo a nadie ni a nada. En tanto, Jovino Novoa y Hernán Larraín insisten en la postura de encontrar responsables políticos a la crisis del Transantiago. Esta mañana, además, el diputado UDI Patricio Melero criticó las explicaciones dadas por Espejo en la sesión especial en Valparaíso: "Lo único que les faltó decir anoche, es que Pinochet es culpable del Transantiago".

Por otra parte, en el Cuadro 2 se puede ver que los actores que mostraron mayor nivel de conflicto fueron el Ministerio de Transporte y los Gremios, y en meno medida el Administrador Financiero del Transantiago (AFT) y los propios usuarios del sistema. Esto puede ser un reflejo de las aristas más controvertidas de la discusión pública. Si bien, por un lado fue evidente la pugna entre el Ministerio y los gremios, los demás porcentajes muestran cómo el AFT -que a pesar de ser el órgano encargado de la recaudación del sistema- concentró las críticas de los usuarios al ser el interlocutor más visible durante las primeras etapas de la implementación. Por último, que hay un porcentaje significativo de las notas más con- 
flictivas asociadas a los usuarios, en un claro reflejo de cómo la política impactó de forma negativa en la población, malestar que también se reveló en los sondeos de opinión pública ${ }^{10}$.

\section{Cuadro 2}

\section{Nivel de conflictividad de las notas publicadas en los diarios de circulación nacional asociadas a un actor (institucional y/o privado)}

\begin{tabular}{|c|c|c|c|c|}
\hline \multirow{2}{*}{ Actor involucrado } & \multicolumn{4}{|c|}{ Nivel de conflictividad } \\
\hline & Muy alta & Alta & Moderada & Baja \\
\hline Ministerio de Transporte & 34,54 & 50,58 & 45,54 & 52,11 \\
\hline Ministerio de Obras Públicas & 0,36 & 0,29 & 0,89 & - \\
\hline Ministerio de Hacienda & 0,90 & 2,60 & 0,45 & 0,70 \\
\hline Ministerio Secretaría General de la Presidencia & 0,90 & 0,87 & 0,45 & 0,70 \\
\hline Gremios & 9,58 & 8,38 & 7,59 & 6,34 \\
\hline Partidos de la Concertación & 2,35 & 3,18 & 2,23 & 0,70 \\
\hline Partidos de Derecha & 6,15 & 2,02 & 1,34 & - \\
\hline Diputados & 2,17 & 2,02 & 3,13 & 0,70 \\
\hline Técnicos & 3,80 & 3,18 & 5,36 & 2,11 \\
\hline Carabineros & 0,72 & - & - & - \\
\hline Administrador Financiero del Transantiago & 9,22 & 5,78 & 7,14 & 1,41 \\
\hline Metro & 3,44 & 0,29 & 0,45 & 3,52 \\
\hline Usuarios & 7,96 & 5,49 & 8,93 & 13,38 \\
\hline Presidencia & 4,52 & 5,49 & 6,25 & 6,34 \\
\hline Grupo Sacyr & 0,18 & 0,29 & - & - \\
\hline Gremio choferes & 4,52 & 2,60 & 0,89 & 2,11 \\
\hline Ministerio Secretaría General de Gobierno & 0,36 & - & 3,57 & - \\
\hline
\end{tabular}

Fuente: Elaboración Propia

10 Según una nota del 3 de febrero de 2008 del Diario El Mercurio: "El Transantiago empeoró salud mental de usuarios. Estrés, malos ratos y problemas laborales influyeron en esta percepción. El $83 \%$ de los usuarios del Transantiago ha visto empeorada su salud mental a pocos días de cumplirse un ańo de la puesta en marcha del sistema de transporte capitalino. Esto, según una encuesta entregada ayer y que fue realizada en conjunto entre Renovación Nacional y la empresa Direct Media. Según el estudio, el 28,14\% de los usuarios le pone nota 1 al actual sistema, el $15,58 \%$ un 2 , el $19,84 \%$ un 3 y el 1,91\% un 7 . Las calificaciones más negativas, sí, tuvieron un leve repunte, en comparación al 31,06\% de diciembre que reprobó con un 1 al Transantiago". 
En esta etapa los partidos políticos aparecen como actores reactivos al proceso, debido a que a su rol se hace visible principalmente en el período de implementación del sistema, cuando se sumaron -junto a otros actores- a las críticas del poco prolijo inicio del sistema. Las críticas se manifestaron en ambas coaliciones, tanto en la centro-derecha, en la Alianza Por Chile, como en la propia Concertación de Partidos por la Democracia ${ }^{11}$. En este sentido, a modo de conclusión previa, podemos observar que la actuación de los actores, como partidos políticos, Congreso y medios de comunicación se presenta habitualmente reactiva a las consecuencias de una política pública, siendo escasa o poco participativa en las etapas previas $^{12}$.

En el cuadro 3 se caracteriza un fenómeno comunicacional, la denominada personalización de los discursos. La personalización es una especie de framing, es decir, un encuadre, que permite enmarcar la información en algunos aspectos noticiosos. Según Dussaillant (2005) "los medios de comunicación han optado por enfocar su contenido desde la perspectiva de las personas más que de los temas o instituciones". Es decir, se recurre a sus características personales más que al hecho o información en sí, seleccionando ciertos elementos de un asunto general. Este concepto se entiende como la presencia en la pauta noticiosa de las personas que ejercen los cargos de poder y se convierten en los "rostros de la noticia", y que en este caso se encarnó principalmente en los ministros sectoriales: Sergio Espejo y René Cortázar,

11 No sólo en la coalición de centro derecha existieron críticas al sistema. Desde la propia Concertación, con el senador del Partido Socialista socialista Camilo Escalona, también se presentó la disidencia: "Siento que el ex Ministro Etcheberry equivocó claramente el cálculo de la flota que necesitaba. No sólo hay problemas de implementación, también hay errores de diseño, o sea existe un conjunto de personas que con la mejor voluntad hicieron cálculos equivocados. Nuestra tecnocracia demostró no saberlo todo sino que comete errores. Aquí hay responsabilidades compartidas" (diario La Nación, 22 de marzo de 2007).

12 Un ejemplo de esta conducta de disidencia ex post se ejemplifica en una nota publicada en el Diario La Tercera, el 7 de febrero de 2008: "Dirigentes de la Alianza interrumpen vacaciones para fustigar primer aniversario de Transantiago. En la madrugada del domingo 10 de febrero se cumplirá un año desde que comenzó a operar el Transantiago. La ocasión propiciará una nueva arremetida de los dirigentes de la Alianza, varios de los cuales interrumpirán sus vacaciones para desplegar una serie de "conmemoraciones" durante el fin de semana. Uno de los críticos más acérrimos al plan de transporte capitalino es el senador gremialista Jovino Novoa, quien regresará desde Nueva York este viernes para afinar los detalles del "festejo" que, en estricto secreto, está organizando su partido para el mediodía de este domingo. En tanto, en Renovación Nacional, en cambio, apostaron por la ironía y ya bautizaron la ocasión como "el cumpleaños negro" del Transantiago. Para tal efecto, habrá globos de ese color y una torta que ilustre las fallas del sistema de transporte capitalino en la actividad que se llevará a cabo el sábado a las 11 de la mañana en Plaza Italia”. 
y en menor medida, de otros actores como los Presidentes Ricardo Lagos y Michelle Bachelet. Aparecen con cierto porcentaje significativo el director ejecutivo del Plan de Transporte Urbano de Santiago, Germán Correa, y Manuel Navarrete, como uno de los representantes de los dueños de autobuses y dirigente gremial del sector.

\section{Cuadro 3}

Nivel de conflictividad de las notas publicadas en los diarios de circulación nacional asociadas a un actor (personaje público)

\begin{tabular}{l|c|c|c|c|c} 
& \multicolumn{5}{c}{ Nivel de conflictividad } \\
\cline { 2 - 5 } Actor asociado a la política & Muy alta & Alta & Moderada & Baja \\
\hline Ricardo Lagos (Presidente) & 4,16 & 2,31 & 1,79 & 3,52 \\
\hline Michelle Bachelet (Presidenta) & 4,88 & 5,78 & 4,91 & 4,23 \\
\hline René Cortázar (Ministro de Transportes) & 15,01 & 25,43 & 29,46 & 8,45 \\
\hline Ministro Sergio Espejo (Ministro de Transportes) & 7,59 & 9,83 & 6,70 & 10,56 \\
\hline Germán Correa (Coordinador Transantiago) & 5,97 & 4,34 & 6,25 & 2,11 \\
\hline Danilo Núnez (Subsecretario de Transportes) & 2,53 & 3,76 & 3,13 & 6,34 \\
\hline Javier Etcheberry (Ministro de Transportes) & 1,81 & 2,60 & 2,68 & 3,52 \\
\hline Jaime Estévez (Ministro de Transportes) & 1,45 & 0,58 & 1,79 & 2,82 \\
\hline Blas Tomic (Presidente de Metro) & 2,35 & 0,87 & 0,45 & 1,41 \\
\hline Clemente Pérez (Presidente de Metro) & 1,63 & 0,58 & 3,57 & 4,23 \\
\hline Manuel Navarrete (dirigente gremial) & 3,25 & 3,18 & 3,13 & 1,41 \\
\hline Héctor Moya (dirigente gremial) & 1,45 & 0,29 & - & - \\
\hline Guillermo Díaz (Subsecretario de Transportes) & 0,18 & 0,87 & - & 4,93 \\
\hline \hline
\end{tabular}

Fuente: Elaboración Propia

En el cuadro 4 podemos observar cómo los dos periódicos de mayor nivel de circulación fueron los que acapararon la mayor cantidad de noticias dedicadas al Transantiago: un 42,4\% de la pauta noticiosa para "El Mercurio" y un 25,8\% para "La Tercera". Mientras, "La Nación", tuvo un 21,2\% de notas sobre el tema. El mayor porcentaje de notas consideradas conflictivas se centró en estos tres medios, con un $46,8 \%$, de la pauta total, lo que comprueba que el Transantiago fue un tema relevante en su pauta noticiosa. 


\section{Cuadro 4}

Desglose de notas, por porcentaje y número de casos según el nivel de conflictividad de las notas publicadas en los distintos medios de circulación nacional

\begin{tabular}{|c|c|c|c|c|c|c|c|c|c|c|}
\hline \multirow{2}{*}{ Medio } & \multicolumn{2}{|c|}{ Muy Alta } & \multicolumn{2}{|c|}{ Alta } & \multicolumn{2}{|c|}{ Moderada } & \multicolumn{2}{|c|}{ Baja } & \multicolumn{2}{|c|}{$\begin{array}{c}\% \text { Total de la } \\
\text { pauta noticiosa }\end{array}$} \\
\hline & $\%$ & $\begin{array}{l}\mathrm{N}^{\circ} \text { de } \\
\text { notas }\end{array}$ & $\%$ & $\begin{array}{l}\mathrm{N}^{\circ} \mathrm{de} \\
\text { notas }\end{array}$ & $\%$ & $\begin{array}{l}\mathrm{N}^{\circ} \mathrm{de} \\
\text { notas }\end{array}$ & $\%$ & $\begin{array}{l}\mathrm{N}^{\circ} \mathrm{de} \\
\text { notas }\end{array}$ & $\%$ & $\begin{array}{l}\mathrm{N}^{\circ} \text { de } \\
\text { notas }\end{array}$ \\
\hline El Mercurio & 46,8 & 259 & 42,2 & 146 & 33,0 & 74 & 40,1 & 57 & 42,4 & 536 \\
\hline La Tercera & 30,4 & 168 & 25,1 & 87 & 21,4 & 48 & 16,2 & 23 & 25,8 & 326 \\
\hline La Segunda & 5,8 & 32 & 7,5 & 26 & 10,7 & 24 & 10,6 & 15 & 7,7 & 97 \\
\hline La Nación & 14,1 & 78 & 22,8 & 79 & 31,3 & 70 & 28,9 & 41 & 21,2 & 268 \\
\hline La Hora & 0,2 & 1 & - & - & - & - & - & - & 0,1 & 1 \\
\hline Otro medio & 2,7 & 15 & 2,3 & 8 & 3,6 & 8 & 4,2 & 6 & 2,9 & 37 \\
\hline Total $n^{\circ}$ notas & & 553 & & 346 & & 224 & & 142 & & 1265 \\
\hline
\end{tabular}

Fuente: Elaboración Propia

$\mathrm{Al}$ analizar el ciclo consenso-disenso desde las publicaciones de prensa, podemos señalar que el contenido conflictivo de las notas estuvo concentrado principalmente en la narración del conflicto durante su período de instalación, y en menor medida en la etapa previa, la formulación. No obstante es particularmente notorio que la hegemonía del discurso de los expertos, la idea de modernización, fuera el eje que se conservó durante todas las etapas del proceso. Un ejemplo claro de la fuerza de este "imaginario social" es la columna de opinión del dirigente democratacristiano Ignacio Walker, entonces Presidente de la Corporación de Estudios para Latinoamérica (CIEPLAN). El texto fue titulado "El coraje de una decisión", y fue publicado el 23 de marzo de 2007 en el diario El Mercurio. En la cual manifiesta su apoyo a la modernización del transporte, definiéndolo como un acto de "coraje necesario":

Hay que armarse de coraje para hacer las llamadas reformas estructurales. Las privatizaciones, la apertura externa, la reforma previsional, en fin, el Transantiago, sólo por citar algunos ejemplos siempre controvertidos, llenos de errores, pero absolutamente necesarios en una perspectiva histórica -y no quiero entrar en la polémica sobre cada una de ellas-, siempre estarán asociadas a grandes trastornos, a ensayos de acierto y error, a costos de distinto tipo, en la vieja y siempre actual lógica de la modernización y las modernizaciones. 
Esta reforma no escapa a esa lógica, y lo que cabe a la autoridad que ha tenido el coraje de decidir e innovar en esta materia es aferrarse al timón, mantener el rumbo, tener la flexibilidad para ajustar lo que haya que ajustar, pero sin titubear ni ceder en lo fundamental. Sobre todo, hay que entender que sector público y sector privado se necesitan mutuamente en el Transantiago, que estamos todos en el mismo barco, y que harían bien tanto el uno como el otro en lograr la máxima coordinación y eficiencia.

\section{CONCLUSIONES}

¿Qué lecciones nos deja este análisis sobre el discurso de una política pública? En un principio comprobar la hipótesis acerca de un imaginario social asociado al predominio de lo técnico, que va mucho más allá de lo que lo fue y para lo que se concibió el plan de transporte público, Transantiago. El análisis de los artículos de prensa, entrevistados y otros trabajos sobre el tema, parecen conjugar la evidencia sobre un discurso hegemónico acerca de lo debían ser estas modernizaciones en los diversos sectores del aparato del Estado. A partir de esta constatación la teoría comunicacional a través de los conceptos como la Espiral del Silencio, nos permite explicar cómo se fue construyendo un discurso mayoritario, o dicho en otras palabras, un "clima de opinión" proclive a la modernización.

En cuanto al rol de los medios, éste parece ser opaco y reactivo, describiendo a través de sus crónicas ese "clima de opinión" instalado por el gobierno y por sus técnicos. Sin bien, en este caso parece no haber disidencias dentro del espectro de los propios periódicos, ni siquiera por una mayor simpatía, cercanía o lejanía partidaria de sus editoriales.

Se hace difícil responder la pregunta de por qué no se generó un espacio para el debate en los medios de comunicación. Si bien la complejidad del tema propuesto (el sistema de transportes) podría ser una respuesta posible, lo cierto, es que eso no es más que una conjetura. Lo cierto es que la evidencia que se traspasa en la lectura de los recortes de prensa, exhibe una pauta que no mostró ideas disidentes (excepto en la postura de los gremios) ni de mayor debate que las opiniones vertidas en sus editoriales o columnas. 
Según lo explican los estudios acerca de las controversias socio-técnicas, el conocimiento técnico puede crear las condiciones para establecer un imaginario social, e incluso, si las condiciones son propensas, transformarlo en un consenso. En esta línea de argumentación, la idea del ciclo del disenso-consenso a la manera que lo describe Elizalde, permite explicar el cambio de estos climas de opinión frente a un discurso público, y cómo, en este caso, el contenido tecnocrático permitió mantener un ciclo de consenso durante la formulación de la política pública.

Percibiendo el fenómeno desde la perspectiva de un gobierno, y particularmente desde el Poder Ejecutivo, la capacidad de mantener el consenso entre los actores de la sociedad estará directamente relacionada con la necesidad de mantener su gobernabilidad. En este caso, los dos períodos del discurso público asociado al Transantiago, manifiestan dos estados de clima social muy distintos. En el primero, la institucionalidad se ve fortalecida por la adopción de un discurso gubernamental compartido por la sociedad. Por el contrario, en la segunda etapa, cuando este relato se ve cuestionado, la salida comunicacional -que nunca puso en cuestión el discurso tecnocrático- se centró en mantener el funcionamiento de las instituciones y buscar a los responsables ${ }^{13}$ de esta implementación poco prolija. Esto es evidente al analizar los diagnósticos acerca del inicio del plan. Algunos hablan acerca de la falta de preparación para la puesta en marcha del plan, a lo que se sumó la falta de presupuesto, aparente improvisación de parte de las autoridades y escasa transparencia en cuanto a los objetivos del plan:

Transantiago nació sin presupuesto, jamás pudo superar esa situación, y su destino quedó determinado por esa condicionante. Las prioridades del gobierno de Ricardo Lagos, como él mismo expresó, eran la construcción de un conjunto de

13 A partir de febrero de 2007, tras el dificultoso inicio del Transantiago, el trabajo académico y también en los medios de comunicación se centró en la búsqueda de las responsabilidades técnicas como políticas. Como ejemplo de este debate son las conclusiones del trabajo de Mardones (2008). "Ningún gobierno puede relativizar su responsabilidad cuando se trata de cumplir misiones de servicio público. Incluso, si éstas se encomiendan al sector privado. En cualquier caso, el gobierno es responsable absoluto de que se ejecuten adecuadamente dichas misiones. (...) La segunda conclusión se refiere a la definición del carácter de una autoridad de transporte. La debilidad de Transantiago no sólo se evidencia en su falta de poder para operar y en su falta de definición para hacer cumplir objetivos como contraparte reguladora, sino, también, en que desde un principio renuncia a cubrir el conjunto de sistemas de transporte de la ciudad y, por ende, el conjunto de territorios contenidos en ella. Una auto-negación de esta naturaleza es bastante presagiadora de lo que se podía esperar. No obstante, si bien esta argumentación había sido desarrollada por técnicos que habían estudiado el sistema desde el interior del gobierno, o bien, en la Academia, la decisión de llevar a cabo la transformación fue esencialmente política”. 
autopistas y la ampliación del Metro, siendo la modernización del sistema de buses un elemento más bien menor, sin grandes aspiraciones, y eso es patente en los recursos asignados; en las autopistas de Santiago se comprometieron dineros fiscales por US \$1.400 millones, en Metro una cifra similar, mientras que a Transantiago se destinaron menos de doscientos millones. Se estableció y mantuvo un discurso intencionalmente falaz, que engañó a la opinión pública aseverando que las autopistas las pagaban sus usuarios, y algo similar para el Metro, al tiempo que se avivaron y explotaron expectativas sobre el Transantiago que, en vista de los recursos disponibles, y los propios antecedentes con que contaba la autoridad, eran absolutamente imposibles de cumplir" (Quijada et al. 2007: 4).

Creemos que los hallazgos de este trabajo nos permiten, en primer lugar, confirmar la idea de la apropiación del saber técnico como una herramienta utilizada para creación de consenso y cómo este discurso hegemónico, asociado a la aspiración de modernización, se enmarcó dentro del programa de gobierno de la centro-izquierda chilena. Lo anterior permitió demostrar ideas como la espiral del silencio, y clima de opinión, y otros de reciente data, como el ciclo de consensodisenso.

Por último, fue posible dar cuenta de que el uso de la perspectiva construccionista a partir de lo que se conoce como controversias socio-técnicas e imaginarios sociales permiten entender el ciclo de la instalación, mantención y quiebre de consenso dentro de un discurso público, y se convierten en parte esencial del proceso de creación de una política pública.

\section{REFERENCIAS BIBLIOGRÁFICAS}

Baeza-Rodríguez, Cecilia (2008). "Los discursos públicos sobre la gobernabilidad en Chile como relatos de acción pública: un enfoque cognitivista sobre la importación de las ideas". Revista web Nuevo Mundo, Mundos Nuevos. Disponible en: http://nuevomundo.revues.org/11042

Pinch T. y Bijker, W, (1989). "The Social Construction of Facts and Artifacts", en W. Bijker, Hughes T. y Pinch T. (eds.), The Social Construction of Technological Systems: New Directions in the Sociology and History of Technology. Cambrigde, MA: MIT Press, pp. 17-50.

Castoriadis, Cornelius (2010). La institución imaginaria de la sociedad. Madrid: Tusquet. 
Cruz, Carlos (2009). "Política de infraestructura desde principios de los noventa: ambición y decepción de la asociación entre públicos y privados”. En Bascunán, Carlos; Correa, Germán Maldonado, Jorge y Sánchez, Vicente (eds.), Más acá de los sueños, más allá de lo posible. La Concertación en Chile. Volumen 2. Santiago: LOM, pp. 191-215.

Cunill, Nuria (1997). Repensando lo público a través de la sociedad. Venezuela: Nueva Sociedad.

Dávila, Mireya (2010). Tecnocracia y democracia en el Chile contemporáneo: el caso de los gobiernos de la Concertación (1990-2010). Revista de Sociología, N²4. pp. 199-217.

Díaz, Guillermo; Gómez-Lobo, Andrés, y Velasco, Andrés (2004). "Micros en Santiago: De enemigo público a servicio público”. Documento de trabajo No 357, Centro de Estudios Públicos.

Dussaillant, Patricio (2005). Medios y Elecciones: La elección presidencial de 1999. Chile: Centro de Estudios Bicentenario, Universidad Los Andes.

Elizalde, Luciano (2006). "La comunicación gubernamental: Problemas y soluciones estratégicas", en Damián Fernández, Luciano Elizalde y Mario Riorda, "La construcción del consenso. Gestión de la comunicación gubernamental”. Buenos Aires: La Crujía Ediciones.

Figueroa, Oscar y Orellana, Arturo (2007). “Transantiago: gobernabilidad e institucionalidad”. Revista EURE, vol. XXXIII, N 100 , pp. 165-171.

Gaete, Andrea (2010). El rol de los grupos de presión en el proceso de formulación de la Política pública denominada Plan AUGE (2000-2006). El caso de los gremios del sector salud. Tesis para optar al grado de Magíster en Ciencia Política. Instituto de Asuntos Públicos. Universidad de Chile.

Garretón, Manuel Antonio (2007). Matriz Sociopolítica y Desarrollo Socio-Económico en Chile. Programme Office, IDPM, School of Environment \& Development, University of Manchester, www.ippg.org.uk. Discussion Paper Series Number Fifteen A.

Jobert, Bruno (2004). Estado, Sociedad, Políticas Públicas. Santiago: LOM.

Jouffe, Yves y Lazo, Alejandra (2010). “Las prácticas cotidianas frente a los dispositivos de la movilidad. Aproximación política a la movilidad cotidiana de las poblaciones pobres periurbanas de Santiago de Chile". Revista EURE, Vol. 36. N 108, pp. 29-47

Kingdon, John (1995). Agendas, alternatives and public policies. USA: University of Michigan.

Lagos, Ricardo (1999). Primer Gobierno del Siglo XXI. Programa de gobierno: para crecer con igualdad. Santiago: Concertación de Partidos por la Democracia.

Latour, Bruno (2005). Reassembling the Social: An introduction to Actor-Network- Theory. UK: Oxford University Press. 
Maillet, Antoine (2008). "La Gestación del Transantiago en el Discurso Público: Hacia un análisis de Políticas Públicas desde la Perspectiva Cognitivista". Revista web Nuevo Mundo, Mundos Nuevos. http://nuevomundo.revues.org/10932

Mardones, Rodrigo (2008). "Chile: Transantiago recargado". Revista de Ciencia Política. Vol. 28, $\mathrm{N}^{\circ}$ 1, pp. 103-119.

Noelle-Neumann, Elisabeth (1995). La espiral del silencio. Opinión pública: nuestra piel social. España: Paidós.

Quijada, R.; Tirachini, A.; Henríquez, R., y Hurtubia, R. (2007). Investigación al Transantiago: Sistematización de Declaraciones hechas ante la Comisión Investigadora, Resumen de Contenidos de los Principales Informes Técnicos, Información de Documentos Públicos Adicionales y Comentarios Críticos. Santiago: Departamento de Ingeniería de Transporte, Universidad de Chile.

Riorda, Mario (2006). "Hacia un modelo de comunicación gubernamental para el consenso" en Fernández, Damián; Elizalde, Luciano y Riorda, Mario (eds.) La construcción del consenso. Gestión de la comunicación gubernamental. Buenos Aires: La Crujía Ediciones, pp. 17-34.

Valle, Luciano (2009). "La construcción del proyecto político” en Bascunán, Carlos; Correa, Germán; Maldonado, Jorge y Sánchez, Vicente (eds.). Más acá de los sueños, más allá de lo posible”. La Concertación en Chile. Volumen 1. Santiago: LOM, pp. 233-247.

Valles, Miguel S. (2007). Técnicas Cualitativas de Investigación Social: Reflexión metodológica y práctica profesional. España: Síntesis.

Yin, Robert (1994). Case study research. Design and methods. Beverly Hills, CA: Sage.

Recibido: 03 septiembre 2011 / Aprobado: 15 noviembre 2011 